\title{
Data-Driven Robust Control of Unknown MIMO Nonlinear System Subject to Input Saturations and Disturbances
}

\author{
Li Wang, ${ }^{1,2}$ Huajun Gong, ${ }^{1}$ and Chunsheng Liu ${ }^{1}$ \\ ${ }^{1}$ College of Automation Engineering, Nanjing University of Aeronautics and Astronautics, Nanjing 210016, China \\ ${ }^{2}$ Nanhang Jincheng College, Nanjing 211156, China \\ Correspondence should be addressed to Li Wang; li-wang1116@163.com
}

Received 15 June 2017; Revised 8 August 2017; Accepted 9 August 2017; Published 10 September 2017

Academic Editor: Asier Ibeas

Copyright (c) $2017 \mathrm{Li}$ Wang et al. This is an open access article distributed under the Creative Commons Attribution License, which permits unrestricted use, distribution, and reproduction in any medium, provided the original work is properly cited.

\begin{abstract}
This paper presented a new data-driven robust control scheme for unknown nonlinear systems in the presence of input saturation and external disturbances. According to the input and output data of the nonlinear system, a recurrent neural network (RNN) data-driven model is established to reconstruct the dynamics of the nonlinear system. An adaptive output-feedback controller is developed to approximate the unknown disturbances and a novel input saturation compensation method is used to attenuate the effect of the input saturation. Under the proposed adaptive control scheme, the uniformly ultimately bounded convergence of all the signals of the closed-loop nonlinear system is guaranteed via Lyapunov analysis. The simulation results are given to show the effectiveness of the proposed data-driven robust controller.
\end{abstract}

\section{Introduction}

Adaptive controls of multi-input and multi-output (MIMO) systems have received significantly increased attention [1-4]. Variables adaptive control techniques have been applied to many practical applications, for example, indirect-adaptive model-following control approach for flying vehicles [5], model reference adaptive control scheme for single-input LTI systems [6], robust control scheme for a class of systems with uncertainty and time delay [7], and adaptive sliding mode scheme for near space vehicles [8], where the established control strategies required some knowledge of nonlinear dynamics and met some specific conditions. However, in most of the practical nonlinear systems, it is difficult to obtain their accurate mathematical models.

Fortunately, input-output data of the nonlinear system can be accessed in many practical control processes. The accessed historical input-output data could be incorporated indirectly in the form of a data-driven model. The data-driven model could be extracted from useful information contained in input-output data, which belongs to the field of data-driven control techniques [9-11]. Then it is desirable to design the controller based on the available input-output data.
In [12], a delayed dynamic neural network was to do online identification for identification problem for time-delay nonlinear system. For the nonlinear MIMO system, a datadriven robust approximate optimal tracking control scheme had been proposed with the aim of resolving the data-driven control problem [13]. In [14], an adaptive dynamic programming was developed to handle zero-sum neuro-optimal control problem for continuous-time unknown nonlinear system with disturbances. However, these controls only considered external disturbances and did not consider input constraints.

Physical input saturation would be encountered in the practical systems and needs to be considered in the designed controls. It may lead to the degradation of system performance, undesirable inaccuracy, or instability. To improve the systems performance, the effect of actuator saturation should be appropriately considered during the control design procedure $[15,16]$. In [17], a dynamic surface control scheme was designed for uncertain strict-feedback nonlinear systems in the presence of input saturation. Adaptive neural network control was investigated for an uncertain nonlinear system with asymmetric saturation actuators [18], where the established control strategies required the known sign of control 
gain and met a specific assumption of uncertain strictfeedback nonlinear system. Although approximation-based adaptive control approaches were proposed for a class of MIMO systems $[19,20]$, the input constraints cannot be compensated when encountering the entirely unknown dynamics model [19]. In [20], the researchers developed an adaptive neural control strategy for a class of affine nonlinear systems.

Motivated by the above-mentioned considerations, this paper focuses on a class of unknown MIMO nonlinear systems with input saturation and external disturbances. In this paper, a data-driven model based on RNN is proposed to reconstruct the unknown system dynamics by using available input-output data. Then a novel robust feedback approximate controller is proposed for the nonlinear system in presence of external disturbance and input saturation via the constructed data-driven model. Radial basis function NN (RBFNN) is employed to tackle the uncertainties and external disturbance, in which the number of online adaptation parameters is reduced to only one, which greatly reduced the computation burden. The integrated control scheme combines adaptive NN robust control with input saturation compensation technique to satisfy the practical requirements in applications.

Compared with the existing literature, the contributions of this brief include the following.

(1) A RNN model is developed to reconstruct the unknown general system dynamics by using inputoutput data of the system, and then a control scheme that integrates adaptive output-feedback control methodologies with data-driven model dynamics is proposed for the unknown MIMO nonlinear systems.

(2) To make the proposed controller more general and practical, the external disturbances, system uncertainties, and the input saturation are considered for the entirely unknown MIMO nonlinear systems in this paper without satisfying the matched parametric uncertainties [16] for a class of affine nonlinear systems [20] or requiring the known sign of the control gain of strict-feedback nonlinear system [18].

The rest of this paper is organized as follows. In Section 2, we present the general problem and the system formulation. An effective RNN model will be established to reconstruct the dynamics of the nonlinear systems. Convergence properties of the weight matrices of RNN will also be presented. In Section 3, the robust controller for unknown nonlinear system with disturbance and input constraints is designed and the stability analysis is developed. Section 4 gives simulation results to verify effectiveness of the proposed method. Finally, the conclusions are drawn in Section 5.

Throughout the paper, the following notations are used.

Notations 1 . $\|\cdot\|$ stands for a suitable norm. When $z$ is a vector, $\|z\|$ denotes the Euclidean norm of $z$. When $z$ is a matrix, $\|z\|$ denotes the two-norm of $z$.

Notations 2. For a given matrix $A, A^{T}$ and $\operatorname{tr}(A)$ represent its transpose and trace, respectively. For a square matrix,
$A>0$ and $A<0$ denote positive-definiteness and negativedefiniteness, respectively.

\section{Problem Formulation and Neurodynamic Model by RNN}

Consider a class of continuous-time nonlinear systems subject to actuator saturation and disturbances in the following form:

$$
\dot{x}(t)=G(x(t), u(t))+\eta_{d},
$$

where $x(t)=\left(x_{1}(t), x_{2}(t), \ldots, x_{n}(t)\right)^{T} \in R^{n}$ is the state vector and $u(t)$ denotes the control input subject to saturation-type nonlinear which is described by $u(t)=\left(u_{1}(t), u_{2}(t), \ldots\right.$, $\left.u_{m}(t)\right)^{T} \in \Xi, \Xi=\left\{u\left|u(t) \in R^{m},\right| u_{i}(t) \mid \leq \alpha_{i}, i=1, \ldots, m\right\}$, where $\alpha_{i}>0$ is the saturating upper bound. The system function $G(\cdot, \cdot)$ is an unknown smooth nonlinear function with respect to $x(t)$ and $u(t)$ on a compact set $\Omega_{x} \subset R^{n}$ containing the origin, and $G(0,0)=0$. Hence, $x=0$ is an equilibrium state of system (1) under the control $u=0 . \eta_{d}$ denotes bounded system external disturbances.

Assumption 3 (see [21-23]). For the disturbances in system (1), there exists unknown positive constant $\bar{\eta}_{d}$ such that $\left\|\eta_{d}\right\| \leq \bar{\eta}_{d}$.

In this paper, in order to tackle the robust control problem for system (1) with input saturation and unknown disturbances, one needs to derive an adaptive feedback control such that the unknown close-loop nonlinear system is globally bounded. Hence, a data-driven method, that is, using the input-output data of unknown system, is desired for the nonlinear system (1). Then, a robust feedback controller based on the RNN model is to be discussed in Section 3 to compensate the nonlinear effect of the disturbances and input saturation constraints. To simplify writing, $u(t)$ and $x(t)$ are abbreviated to $u$ and $x$ in some subsequent formulas, respectively.

Remark 4. During reconstructing the RNN model, the external disturbances are considered; the system uncertainty and input saturation compensation problem is solved in Section 3.

The continuous-time nonlinear system dynamics (1) can be reconstructed in the form of an RNN as follows:

$$
\dot{x}=A^{*} x+C^{*} f(x)+B^{*} u(t)+D^{*}+\eta_{d}+\eta_{x},
$$

where $A^{*}, B^{*}, C^{*}$, and $D^{*}$ are the unknown ideal weight matrices. $\eta_{x}$ denotes a finite approximate error, which satisfies $\left\|\eta_{x}\right\| \leq \bar{\eta}_{x}$, where $\bar{\eta}_{x}$ is positive number. From Assumption 3, thus $\eta=\eta_{d}+\eta_{x}$ is taken as the compound disturbance which satisfies $\|\eta\| \leq \bar{\eta}=\bar{\eta}_{d}+\bar{\eta}_{x}$.

Assumption 5 (see [13]). The norm of matrices $A^{*}, B^{*}, C^{*}$, and $D^{*}$ is assumed to be bounded, which satisfied $\left\|A^{*}\right\| \leq \bar{A}$, $\left\|B^{*}\right\| \leq \bar{B},\left\|C^{*}\right\| \leq \bar{C}$, and $\left\|D^{*}\right\| \leq \bar{D}$, where $\bar{A}, \bar{B}, \bar{C}$, and $\bar{D}$ are all positive constants. 
Assumption 6 (see [24]). The activation function $f(x)$ is a given local Lipschitz continuous differentiable function; that is, a function $f(x):[a, b] \rightarrow R$ is local Lipschitz on $[a, b]$, $\left\|f\left(x_{1}\right)-f\left(x_{2}\right)\right\| \leq k\left|x_{1}-x_{2}\right|$ for all $x_{1}, x_{2} \in[a, b]$, where $k>0$ is a positive constant.

Selection of active function $f(x)$ would guarantee that the controller exists and is obtainable by dichotomy principle.

Based on (2), the data-driven RNN model can be constructed as

$$
\dot{\hat{x}}=\widehat{A} x+\widehat{B} u+\widehat{C} f(\widehat{x})+\widehat{D}+\varepsilon e_{x},
$$

where $\widehat{A}, \widehat{B}, \widehat{C}$, and $\widehat{D}$ are the estimated weight matrices of the ideal unknown weight matrices $A^{*}, B^{*}, C^{*}$, and $D^{*}$, respectively. Define the weight estimation error matrices as $\widetilde{A}=A^{*}-\widehat{A}, \widetilde{B}=B^{*}-\widehat{B}, \widetilde{C}=C^{*}-\widehat{C}, \widetilde{D}=D^{*}-\widehat{D}$ and state estimation error as $e_{x}=x-\widehat{x}, \widetilde{f}\left(e_{x}\right)=f(x)-f(\widehat{x})$.

Considering Assumption 6, let $\varepsilon$ in (3) be a square matrix that satisfies

$$
\lambda_{\min }\left(\varepsilon-A^{*}-\frac{1}{2} C^{*} C^{* T}\right) \geq \frac{1}{2} k^{2}+\bar{\eta}
$$

where $\lambda_{\min }(\cdot)$ is the representation of the minimum eigenvalue.

The state estimation error $e_{x}$ derivative is obtained from (2) and (3), which is given below:

$$
\begin{aligned}
\dot{e}_{x}= & \dot{x}-\dot{\hat{x}} \\
= & A^{*} x+C^{*} f(x)+B^{*} u+\widetilde{D}+\eta-\widehat{A} \widehat{x}-\widehat{C} f(\widehat{x}) \\
& -\widehat{B} u-\varepsilon e_{x} .
\end{aligned}
$$

According to the equations

$$
\begin{aligned}
A^{*} x-\widehat{A} \hat{x} & =A^{*} e_{x}+\widetilde{A} \widehat{x}, \\
C^{*} f(x)-\widehat{C} f(\widehat{x}) & =C^{*} \widetilde{f}\left(e_{x}\right)+\widetilde{C} f(\widehat{x}), \\
B^{*} u-\widehat{B} u & =\widetilde{B} u,
\end{aligned}
$$

the derivative of $e_{x}$ is

$$
\begin{aligned}
\dot{e}_{x}= & A^{*} e_{x}+\widetilde{A} \widehat{x}+C^{*} f\left(e_{x}\right)-\widetilde{C} f(\widehat{x})+\widetilde{B} u+\widetilde{D}+\eta \\
& -\varepsilon e_{x} .
\end{aligned}
$$

Theorem 7. Consider the RNN model system (3), let Assumption 9 hold, and the adaptive update rules of the estimated weight matrices $\widehat{A}, \widehat{B}, \widehat{C}, \widehat{D}$ in (3) can be expressed as

$$
\begin{aligned}
& \dot{\hat{A}}^{T}=\tau_{A} \widehat{x} e_{x}^{T}-\tau_{A} \widehat{A}^{T}, \\
& \dot{\hat{B}}^{T}=\tau_{B} u e_{x}^{T}-\tau_{B} \widehat{B}^{T}, \\
& \dot{\vec{C}}^{T}=\tau_{C} f(\widehat{x}) e_{x}^{T}-\tau_{C} \widehat{C}^{T}, \\
& \dot{\vec{D}}^{T}=\tau_{D} e_{x}^{T}-\tau_{D} \widehat{D}^{T},
\end{aligned}
$$

where the learning rate parameters $\tau_{A}, \tau_{B}, \tau_{C}$, and $\tau_{D}$ are all positive design constants.

Then the adaptive update rules (8)-(11) can guarantee that the uniformly ultimate boundedness of identification estimation error $e_{x}$ and the weight estimation error matrices $\widetilde{A}, \widetilde{B}, \widetilde{C}, \widetilde{D}$ are all $U U B$.

Proof. Define Lyapunov function:

$$
V_{1}=V_{x}+V_{A}+V_{B}+V_{C}+V_{D}
$$

where $V_{x}=(1 / 2) e_{x}{ }^{T} e_{x}, V_{A}=\left(1 / 2 \tau_{A}\right) \operatorname{tr}\left(\widetilde{A}^{T} \widetilde{A}\right), V_{B}=$ $\left(1 / 2 \tau_{B}\right) \operatorname{tr}\left(\widetilde{B}^{T} \widetilde{B}\right), V_{C}=\left(1 / 2 \tau_{C}\right) \operatorname{tr}\left(\widetilde{C}^{T} \widetilde{C}\right)$, and $V_{D}=$ $\left(1 / 2 \tau_{D}\right) \widetilde{D}^{T} \widetilde{D}$.

Differentiating $V_{1}$ and invoking (8)-(11) and (12), we get

$$
\begin{aligned}
\dot{V}_{x}= & e_{x}{ }^{T} A^{*} e_{x}+e_{x}{ }^{T} C^{*} \widetilde{f}\left(e_{x}\right)+e_{x}^{T} \widetilde{A} \widehat{x}+e_{x}{ }^{T} \widetilde{C} f(\widehat{x}) \\
& +e_{x}^{T} \widetilde{B} u+e_{x}{ }^{T} \widetilde{D}+e_{x}{ }^{T} \eta-e_{x}{ }^{T} \varepsilon e_{x} \\
\dot{V}_{A}= & -\operatorname{tr}\left(\widehat{x} e_{x}^{T} \widetilde{A}\right)+\operatorname{tr}\left(\widehat{A}^{T} \widetilde{A}\right) \\
\dot{V}_{B}= & -\operatorname{tr}\left(u e_{x}{ }^{T} \widetilde{B}\right)+\operatorname{tr}\left(\widehat{B}^{T} \widetilde{B}\right) \\
\dot{V}_{C}= & -\operatorname{tr}\left(f(\widehat{x}) e_{x}^{T} \widetilde{C}\right)+\operatorname{tr}\left(\widehat{C}^{T} \widetilde{C}\right) \\
\dot{V}_{D}= & -e_{x}{ }^{T} \widetilde{D}+\widehat{D}^{T} \widetilde{D}
\end{aligned}
$$

where $\dot{\widetilde{A}}=-\dot{\widehat{A}}, \dot{\widetilde{B}}=-\dot{\widehat{B}}, \dot{\widetilde{C}}=\dot{\widehat{C}}, \dot{\widetilde{D}}=-\dot{\widehat{D}}$.

Let $\overline{\bar{A}}=(1 / 2) \operatorname{tr}\left(A^{* T} A^{*}\right)-(1 / 2) \operatorname{tr}\left(\widehat{A}^{T} \widehat{A}\right), \overline{\bar{C}}=$ $(1 / 2) \operatorname{tr}\left(C^{* T} C^{*}\right)-(1 / 2) \operatorname{tr}\left(\widehat{C}^{T} \widehat{C}\right), \overline{\bar{B}}=(1 / 2) \operatorname{tr}\left(B^{* T} B^{*}\right)-$ $(1 / 2) \operatorname{tr}\left(\widehat{B}^{T} \widehat{B}\right), \overline{\bar{D}}=(1 / 2) D^{* T} D^{*}-(1 / 2) \widehat{D}^{T} \widehat{D}, \tau=\overline{\bar{A}}+\overline{\bar{B}}+$ $\overline{\bar{C}}+\overline{\bar{D}}$ be unknown constants.

Then, we have

$$
\begin{aligned}
& \dot{V}_{A}=-\operatorname{tr}\left(\widehat{x} e_{x}^{T} \widetilde{A}\right)-\frac{1}{2} \operatorname{tr}\left(\widetilde{A}^{T} \widetilde{A}\right)+\overline{\bar{A}}, \\
& \dot{V}_{B}=-\operatorname{tr}\left(u e_{x}^{T} \widetilde{B}\right)-\frac{1}{2} \operatorname{tr}\left(\widetilde{B}^{T} \widetilde{B}\right)+\overline{\bar{B}}, \\
& \dot{V}_{C}=-\operatorname{tr}\left(f(\widehat{x}) e_{x}^{T} \widetilde{C}\right)-\frac{1}{2} \operatorname{tr}\left(\widetilde{C}^{T} \widetilde{C}\right)+\overline{\bar{C}}, \\
& \dot{V}_{D}=-e_{x}^{T} \widetilde{D}-\frac{1}{2} \widetilde{D}^{T} \widetilde{D}+\overline{\bar{D}} .
\end{aligned}
$$

According to Assumption 6, we have

$$
\begin{aligned}
e_{x}{ }^{T} C^{*} \tilde{f}\left(e_{x}\right) & \leq \frac{1}{2} e_{x}{ }^{T} C^{*} C^{* T} e_{x}+\frac{1}{2} \tilde{f}\left(e_{x}\right)^{T} \tilde{f}\left(e_{x}\right) \\
& \leq \frac{1}{2} e_{x}{ }^{T} C^{*} C^{* T} e_{x}+\frac{1}{2} k^{2}\left\|e_{x}\right\|^{2} .
\end{aligned}
$$


Substituting (13) and (18)-(19) in (12) gives

$$
\begin{aligned}
\dot{V}_{1}= & e_{x}{ }^{T} A^{*} e_{x}+e_{x}{ }^{T} C^{*} \widetilde{f}\left(e_{x}\right)+e_{x}{ }^{T} \eta_{d}-e_{x}{ }^{T} \varepsilon e_{x} \\
& -\frac{1}{2} \operatorname{tr}\left(\widetilde{A}^{T} \widetilde{A}\right)-\frac{1}{2} \operatorname{tr}\left(\widetilde{B}^{T} \widetilde{B}\right)-\frac{1}{2} \operatorname{tr}\left(\widetilde{C}^{T} \widetilde{C}\right) \\
& -\frac{1}{2} \widetilde{D}^{T} \widetilde{D}+\tau \\
\leq & -\left(\lambda_{\min }(N)-\frac{1}{2}\left(k^{2}+\bar{\eta}\right)\right) e_{x}{ }^{T} e_{x}-\frac{1}{2} \operatorname{tr}\left(\widetilde{A}^{T} \widetilde{A}\right) \\
& -\frac{1}{2} \operatorname{tr}\left(\widetilde{B}^{T} \widetilde{B}\right)-\frac{1}{2} \operatorname{tr}\left(\widetilde{C}^{T} \widetilde{C}\right)-\frac{1}{2} \widetilde{D}^{T} \widetilde{D}+\tau
\end{aligned}
$$

where $N=\varepsilon-A^{*}-\left(C^{*} C^{* T}\right) / 2$ and $\lambda_{\min }(N)$ is minimum eigenvalue of the matrix $N$.

Using (20), we can see if the designed square matrix $\varepsilon$ satisfies

$$
\kappa=\lambda_{\text {min }}(N)-\left(\frac{k^{2}+\bar{\eta}}{2}\right)>0 .
$$

Then all of the signals such as $e_{x},\|\widetilde{A}\|,\|\widetilde{B}\|,\|\widetilde{C}\|$, and $\|\widetilde{D}\|$ are guaranteed UUB properties. The proof is completed.

Remark 8. According to Theorem 7, in unknown continuous-time nonlinear case of this paper, we can see that the RNN-based system state $\widehat{x}(t)$ will converge to the ideal state $x(t)$ when $t \rightarrow \infty$. The update rules of weight matrices $\widehat{A}$, $\widehat{B}, \widehat{C}$, and $\widehat{D}$ are all in continuous-time domain and $\widehat{A}, \widehat{B}, \widehat{C}$, and $\widehat{D}$ will converge to the ideal matrices $A^{*}, B^{*}, C^{*}$, and $D^{*}$, respectively. Thus, it is a merit of the developed method.

\section{Robust Control Based-NN for Unknown Nonlinear System Disturbance and Input Saturation}

The control objective is that the unknown continuoustime nonlinear system with input saturation and disturbance can be stable under the developed adaptive feedback control scheme. Based on data-driven control theories, the continuous-time adaptive law of weight matrices $\widehat{A}, \widehat{B}, \widehat{C}$, and $\widehat{D}$ is preferred. Thus, the original robust control problem of unknown continuous-time nonlinear system (1) is transformed into the robust control for system (2); for example, it has been transformed to design the stable controller for the reconstruction models based-RNN. The detailed robust control scheme and the corresponding parameters updating are presented in this section.

Consequently, the nonlinear system (2) can be rewritten as

$$
\dot{x}=A x+\Delta A(x(t))+B u(t),
$$

where $x \in R^{n}, A, B, C, D$ are the output of RNN-based system corresponding state vector and steady weight matrices. $\Delta A(x(t))=C f(x)+\vartheta$ is the compound disturbance, in which $\vartheta=\left(A^{*}-A\right) x+\left(B^{*}-B\right) u+\left(C^{*}-C\right) f(x)+D^{*}-D+\eta_{x}+$ $\eta_{d}(t)$ can be seen as a finite uncertainty vector which includes the modeling error, approximation error, the perturbation of aerodynamic coefficients, aerodynamic moment coefficients, and external time-varying unknown atmospheric turbulence; thus, $\Delta A(x(t))$ can be seen as a finite compound disturbance vector. The $u(v)=\left[u\left(v_{1}\right), u\left(v_{2}\right), \ldots, u\left(v_{m}\right)\right]^{T} \in R^{m}$ denotes the plant input vector subjected to saturation nonlinearity, which is constrained and defined as

$$
u\left(v_{i}\right)=\operatorname{sat}\left(v_{i}(t)\right)=\operatorname{sgn}\left(v_{i}(t)\right) \cdot \min \left(\alpha_{i},\left|v_{i}(t)\right|\right),
$$

where $v=\left[v_{1}, \ldots, v_{m}\right]^{T} \in R^{m}$ is the system virtual control input vector to be designed later. $\alpha_{i}>0$ is the known saturation parameter of $u\left(v_{i}\right)$, which comes either from a physical process constraint or from an artificial limiter. $\operatorname{sgn}(\cdot)$ denotes the sign of function $(\cdot) \cdot \min (\cdot, \cdot)$ denotes the minimum of $(\cdot)$ and $(\cdot)$. Clearly, between the applied actual control $u(v(t))$ and the desired control input $v(t)$, there is difference. To facilitate the controller design later, define following function:

$$
\Delta(v)=u-v .
$$

Assumption 9. The input difference $\Delta(v)$ between the saturation input $u(t)$ and the desired control input $v(t)$ is bounded with an unknown bound; that is, $r=\|\Delta(v)\| \leq \bar{\Delta}$.

With the bounded property of the saturation input and desired input, we can see that the difference $\Delta(v)$ is bounded, and Assumption 9 is reasonable.

System (22) can be rewritten as

$$
\dot{x}=A x+\Delta A(x, t)+B v+B \Delta(v) .
$$

The control objective is now to design an adaptive feedback controller for system (25) as well as for nonlinear system (1). A baseline control law $v(t)$ based on the indirectadaptive control method is developed to ensure that the closed-loop system is stable.

Lemma 10 (see [25]). The following inequality holds for any $\sigma_{0}>0$ and $\varsigma \in R$ :

$$
0 \leq|\varsigma|-\varsigma \tanh \left(\frac{\varsigma}{\sigma_{0}}\right) \leq \kappa \sigma_{0},
$$

where $\kappa$ is a constant satisfying $\kappa=e^{-(\kappa+1)}$; that is, $\kappa=0.2785$.

Lemma 11 (see [26]). For bounded initial conditions, if there exist a $C^{1}$ continuous and positive definite Lyapunov function $V(x)$ satisfying $a(\|x\|) \leq V(x) \leq b(\|x\|)$ such that $\dot{V}(x) \leq$ $-\delta V(x)+\pi$, where $a, b: R^{n} \rightarrow R$ are class $K$ functions and $\delta, \pi$ are positive constants, then the solution $x(t)$ is uniformly ultimately bounded (UUB).

Equation (25) can be rewritten as

$$
\dot{x}=-x+B E x+B v+g(x)+B \Delta(v),
$$

where $E \in R^{m \times n}$ is an appropriately dimension designed matrix which satisfied that the matrix $A+I_{n \times n}-B E$ is Hurwitz 
matrix. $g(x)=A x+\Delta A(x, t)+x-B E x, g(x)$ is an unknown continuous equivalent disturbance.

In many references of robust adaptive control engineering [27-29], NN was usually employed as approximation tool for modeling continuous nonlinear function term because of its good approximation capability. In this paper, a radial basis function neural network (RBFNN) is used to approximate the unknown equivalent disturbance $g(x)$. Under the optimal weight value, the unknown term can be expressed as

$$
g(x)=B\left(W^{* T} \psi(x)+\xi(t)\right), \quad\|\xi(t)\| \leq \xi_{m},
$$

where $W^{*}$ is the optimal weight value in the approximation. $\xi(t)$ is the smallest approximation error; $\xi_{m}$ is an upper bound of the approximation error $\xi(t)$.

Substituting (28) into (27) yields

$$
\dot{x}=-x+B E x+B v+B W^{* T} \psi(x)+B \xi+B \Delta(v) .
$$

Remark 12. The conventional adaptive $\mathrm{NN}$ approach is used to estimate the weight matrix $W^{*}$ themselves. In this paper, we introduce a novel unknown constant $\omega$ which is specified as $\theta=\left[\begin{array}{llll}\theta_{1} & \theta_{2} & \cdots & \theta_{n}\end{array}\right]^{T}, \theta_{i}=\left\|W_{* i}{ }^{*}\right\|^{2}, i=1,2, \ldots, n, \omega=$ $\sum \theta_{i}$. In this way, the presented adaptive law of NN contains only one parameter regardless of the order of systems. Therefore, the number of adaptation laws of $\mathrm{NN}$ is reduced considerably, which successfully handles this "dimensionality curse" problem.

Doing some simple mathematical manipulation, we have

$$
\begin{aligned}
\chi W^{* T} \psi & =\sum_{i=1}^{m} \chi_{i} W_{i *}^{* T} \psi \leq \frac{1}{2} \sum_{i=1}^{m} \chi_{i}^{2} \theta_{i} \psi^{T} \psi+\frac{1}{2} \\
& \leq \frac{1}{2}\|\chi\|^{2} \omega \psi^{T} \psi+\frac{1}{2},
\end{aligned}
$$

where $\chi=\left(x^{T} B\right)^{T} \in R^{m}$.

Thus, we can obtain

$$
-\frac{1}{2}\|\chi\|^{2} \widehat{\widehat{\omega}} \psi^{T} \psi+\chi W^{* T} \psi \leq \frac{1}{2}\|\chi\|^{2} \widetilde{\omega} \psi^{T} \psi+\frac{1}{2},
$$

where $\widehat{\omega}$ is the estimation of $\Phi, \widetilde{\omega}=\Phi-\widehat{\omega}$.

Design the virtual input controller:

$$
\begin{aligned}
v= & -E x-\tanh \left(\frac{\chi^{T}}{\omega_{0}}\right) \widehat{r}-\tanh \left(\frac{\chi^{T}}{\sigma_{0}}\right) \widehat{\xi}_{m} \\
& -\frac{1}{2} \chi^{T} \widehat{\omega} \psi^{T} \psi
\end{aligned}
$$

where $\widehat{r}$ is the estimator of $r$.

Substituting controller (32) into system (29),

$$
\begin{aligned}
\dot{x}= & -x-B \tanh \left(\frac{\chi^{T}}{\omega_{0}}\right) \widehat{r}-B \tanh \left(\frac{\chi^{T}}{\sigma_{0}}\right) \widehat{\xi}_{m}+B \xi \\
& +B W^{* T} \psi(x)-\frac{1}{2} B \chi^{T} \widehat{\widehat{\omega}} \psi^{T} \psi+B \Delta(v) .
\end{aligned}
$$

Design the following adaptation estimation algorithm of unknown parameter:

$$
\begin{aligned}
& \dot{\vec{\omega}}=\|\chi\|^{2} \psi^{T} \psi+\delta_{\varrho} \widehat{\omega}, \\
& \dot{\hat{r}}=\chi \tanh \left(\frac{\chi^{T}}{\omega_{0}}\right)+\delta_{r} \widehat{r}, \\
& \dot{\vec{\xi}}_{m}=\chi \tanh \left(\frac{\chi^{T}}{\sigma_{0}}\right)+\delta_{\xi_{m}} \widehat{\xi}_{m},
\end{aligned}
$$

where $\delta_{\emptyset}>0, \delta_{r}>0$, and $\delta_{\xi_{m}}>0$ are the small design positive constants, modification terms which are introduced to improve the robustness in the presence of the estimate error. $\omega_{0}, \sigma_{0}$ are design positive constants.

It can be seen that the adapting law of $\mathrm{NN}$ was designed by the unknown parameter $\widehat{\widehat{\omega}}$ not the estimation parameter of weight matrix $\widehat{W}$ in (34). By the adaptive law of NN, the term $B W^{* T} \psi(x)$ of (33) was to be offset, that is, the unknown equivalent disturbance $g(x)$ of (27) was to be compensated.

Remark 13. From (36), we can see that the known upper boundary requirement of the unknown NN approximation error is eliminated for the design adaptation law of estimation of NN approximation error.

Theorem 14. Consider the unknown MIMO nonlinear system (1) with input constraints and the unknown external disturbance. The update law of the weight matrix of RNN model is designed in (8), (9), (10), and (11), respectively. The virtual input control law and parameter tuning laws are designed as (32), (34), (35), and (36), respectively. Then, by selecting the design parameters appropriately, the designed control scheme can guarantee that all signals in the closed-loop system are bounded; for example, the signals $x, \widetilde{\omega}, \widetilde{r}$, and $\widetilde{\xi}_{m}$ are uniformly ultimately bounded.

Proof. Consider the following Lyapunov function candidate:

$$
L=\sum_{i=1}^{2} L_{i},
$$

where $L_{1}=\left(x^{T} x\right) / 2, L_{2}=\left(\widetilde{\omega}^{2}\right) / 4+\left(\widetilde{r}^{2}\right) / 2+\left(\widetilde{\xi}_{m}^{2}\right) / 2$.

Let $\widetilde{r}=r-\widehat{r}, \widetilde{\omega}=\omega-\widehat{\omega}$. Invoking (33), the time derivative of $L_{1}$ is given by

$$
\begin{aligned}
\dot{L}_{1}= & -x^{T} x-\chi \tanh \left(\frac{\chi^{T}}{\omega_{0}}\right) \widehat{r}-\chi \tanh \left(\frac{\chi^{T}}{\sigma_{0}}\right) \widehat{\xi}_{m} \\
& -\frac{1}{2} \chi \chi^{T} \widehat{\widehat{\omega}} \psi^{T} \psi+\chi W^{* T} \psi(x)+\chi \xi+\chi \Delta(v) .
\end{aligned}
$$

From (30), the time derivate of $L_{1}$ can be rewritten as

$$
\begin{aligned}
\dot{L}_{1} \leq & -x^{T} x-\chi \tanh \left(\frac{\chi^{T}}{\omega_{0}}\right) \widehat{r}-x^{T} B \tanh \left(\frac{\chi^{T}}{\sigma_{0}}\right) \widehat{\xi}_{m} \\
& -\frac{1}{2}\|\chi\|^{2} \widetilde{\omega} \psi^{T} \psi+\|\chi\|_{1} \xi_{m}+\|\chi\|_{1} r+\frac{1}{2} .
\end{aligned}
$$


Using Lemma 10, we can obtain

$$
\begin{aligned}
& \|\chi\|_{1} r-\chi \tanh \left(\frac{\chi^{T}}{\omega_{0}}\right) r \\
& \quad=r\left(\sum_{i=1}^{n}\left|\chi_{i}\right|-\sum_{i=1}^{n} \chi_{i} \tanh \left(\frac{\chi_{i}}{\omega_{0}}\right)\right) \leq 0.2785 n r \omega_{0}, \\
& \|\chi\|_{1} \xi_{m}-\chi \tanh \left(\frac{\chi^{T}}{\sigma_{0}}\right) \xi_{m} \\
& \quad=\xi_{m} \sum_{i=1}^{n}\left(\left|\chi_{i}\right|-\chi_{i} \tanh \left(\frac{\chi_{i}}{\sigma_{0}}\right)\right) \leq \xi_{m} \sum_{i=1}^{n} 0.2785 \sigma_{0} \\
& =0.2785 n \sigma_{0} \xi_{m} .
\end{aligned}
$$

From (40), the time derivative of $L_{1}$ is given by

$$
\begin{aligned}
\dot{L}_{1} \leq & -x^{T} x+\chi \tanh \left(\frac{\chi^{T}}{\omega_{0}}\right) \widetilde{r}+\chi \tanh \left(\frac{\chi^{T}}{\sigma_{0}}\right) \widetilde{\xi}_{m}+\frac{1}{2} \\
& +\frac{1}{2}\|\chi\|^{2} \widetilde{\omega} \psi^{T} \psi \\
& +\sum_{i=1}^{n}\left(0.2785 r \omega_{0}+0.2785 \xi_{m} \sigma_{0}\right) .
\end{aligned}
$$

From (37), the time derivate of $L_{2}$ is

$$
\dot{L}_{2}=-\frac{1}{2} \dot{\widehat{\hat{\omega}}} \widetilde{\omega}-\dot{\hat{r}} \tilde{r}-\dot{\hat{\xi}}_{m} \widetilde{\xi}_{m}
$$

where $\dot{\widetilde{\widehat{\omega}}}=-\dot{\widehat{\widehat{\omega}}}, \dot{\vec{r}}=-\dot{\hat{r}}, \dot{\vec{\xi}}_{m}=-\dot{\vec{\xi}}_{m}$.

Substituting the parameter updating laws (34)-(36) into (42), we have the following equality:

$$
\begin{aligned}
\dot{L}_{2}= & -\frac{1}{2}\|\chi\|^{2} \psi^{T} \psi \widetilde{\omega}-\frac{1}{2} \delta_{\widehat{\omega}} \widehat{\omega} \widetilde{\omega}-\chi \tanh \left(\frac{\chi^{T}}{\omega_{0}}\right) \widetilde{r} \\
& -\delta_{r} \widetilde{r} \widetilde{r}-\chi \tanh \left(\frac{\chi^{T}}{\sigma_{0}}\right) \widetilde{\xi}_{m}-\delta_{\xi_{m}} \widehat{\xi}_{m} \widetilde{\xi}_{m} .
\end{aligned}
$$

The following inequalities hold

$$
\begin{aligned}
-\frac{1}{2} \delta_{\omega} \widehat{\omega} \widetilde{\omega} & \leq-\frac{1}{4} \delta_{\omega} \widetilde{\omega}^{2}+\frac{1}{4} \delta_{\omega} \omega^{2}, \\
-\delta_{r} \widetilde{r} \widetilde{r} & \leq-\frac{1}{2} \delta_{r} \widetilde{r}^{2}+\frac{1}{2} \delta_{r} r^{2}, \\
-\delta_{\xi_{m}} \widehat{\xi}_{m} \widetilde{\xi}_{m} & \leq-\frac{1}{2} \delta_{\xi_{m}} \widetilde{\xi}_{m}{ }^{2}+\frac{1}{2} \delta_{\xi_{m}} \xi_{m}{ }^{2} .
\end{aligned}
$$
ity:

Substituting (44) into (43), we have the following inequal-

$$
\begin{aligned}
\dot{L}_{2} \leq & -\frac{1}{2}\|\chi\|^{2} \psi^{T} \psi \widetilde{\omega}-\frac{1}{4} \delta_{\varpi}\|\widetilde{\omega}\|^{2}+\frac{1}{4} \delta_{\emptyset}\|\omega\|^{2} \\
& -\chi \tanh \left(\frac{\chi^{T}}{\omega_{0}}\right) \widetilde{r}-\frac{1}{2} \delta_{r} \widetilde{r}^{2}+\frac{1}{2} \delta_{r} r^{2} \\
& -\chi \tanh \left(\frac{\chi^{T}}{\sigma}\right) \widetilde{\xi}_{m}-\frac{1}{2} \delta_{\xi_{m}} \widetilde{\xi}_{m}{ }^{2}+\frac{1}{2} \delta_{\xi_{m}} \xi_{m}{ }^{2} .
\end{aligned}
$$

From (39) and (45), we have

$$
\begin{aligned}
\dot{L} \leq & -x^{T} x-\frac{1}{4} \delta_{\varpi} \widetilde{\omega}^{2}-\frac{1}{2} \delta_{r} \widetilde{r}^{2}+\frac{1}{2}-\frac{1}{2} \delta_{\xi_{m}} \widetilde{\xi}_{m}^{2} \\
& +\frac{1}{4} \delta_{\omega} \omega^{2}+\frac{1}{2} \delta_{\xi_{m}} \xi_{m}{ }^{2}+\frac{1}{2} \delta_{r} r^{2} \\
& +n 0.2785\left(r \omega_{0}+\xi_{m} \sigma_{0}\right) .
\end{aligned}
$$

Then we have the following conclusion:

$$
\dot{L} \leq-\beta L+\varphi,
$$

where $\beta=\min \left(1, \delta_{\varpi} / 4, \delta_{r} / 2, \delta_{\xi_{m}} / 2\right), \varphi=\delta_{\xi_{m}} \xi_{m}{ }^{2} / 2+$ $\delta_{\omega} \omega^{2} / 4+\delta_{r} r^{2} / 2+1 / 2+n 0.2785\left(r \omega_{0}+\xi_{m} \sigma_{0}\right), \varphi$ and $\beta$ are positive constants.

Finally, we have

$$
L \leq \frac{\varphi}{\beta}+\vartheta e^{-\beta t}
$$

where $\vartheta=L(0)-\varphi / \beta, L=f\left(x, \widetilde{\omega}, \widetilde{r}, \widetilde{\xi}_{m}\right)$. Thus, $\lim _{t \rightarrow \infty} L(t) \leq$ $\varphi / \beta, \forall t>0$.

With the help of Lemma 11, $x, \widetilde{\omega}, \widetilde{r}, \widetilde{\xi}_{m}$ are all uniformly ultimately bounded (UUB). This proof is completed.

From Theorems 7 and 14, it is noted that the weight matrices adaptation laws (8)-(11) during the process of modeling and the designed controller (32) guaranteed that the closed-loop system (1) is UUB.

\section{Simulation}

To evaluate the performance of our algorithm, we give two examples for the numerical experiments.

Example 15. Consider the following complex affine nonlinear continuous-time system:

$$
\begin{aligned}
\dot{x}_{1}= & -x_{1}+x_{2}+2 x_{2}^{3}+d(t), \\
\dot{x}_{2}= & -0.5\left(x_{1}+x_{2}\right) x_{1}^{2}+0.5 x_{2}\left(1+2 x_{2}^{2}\right) \sin \left(x_{1}\right)^{2} \\
& +u .
\end{aligned}
$$

It is assumed that the system dynamics is unknown and input-output data are available. The external disturbance signal $d(t)$ was chosen as

$$
d(t)=\operatorname{rand}(-1,1) e^{-0.2 t} \cos (t),
$$

where $\operatorname{rand}(-1,1)$ denotes the random number in $[-1,1]$. Except for the external disturbance, the input constraint is added to system (49).

The activation function $f(\hat{x})$ is selected as hyperbolic tangent function $\tanh (\hat{x})$. Let the $\mathrm{RNN}$ with the initial elements of matrices $\widehat{A}, \widehat{B}, \widehat{C}, \widehat{D}$ be randomly selected in $[-0.5,1]$. Select the design parameter in Theorem 7 as $\tau_{A}=$ $\tau_{B}=\tau_{C}=\tau_{D}=0.9$. The design gain matrix $\varepsilon=\left[\begin{array}{llll}1 & 6 ; & -5 & 8\end{array}\right]$. 


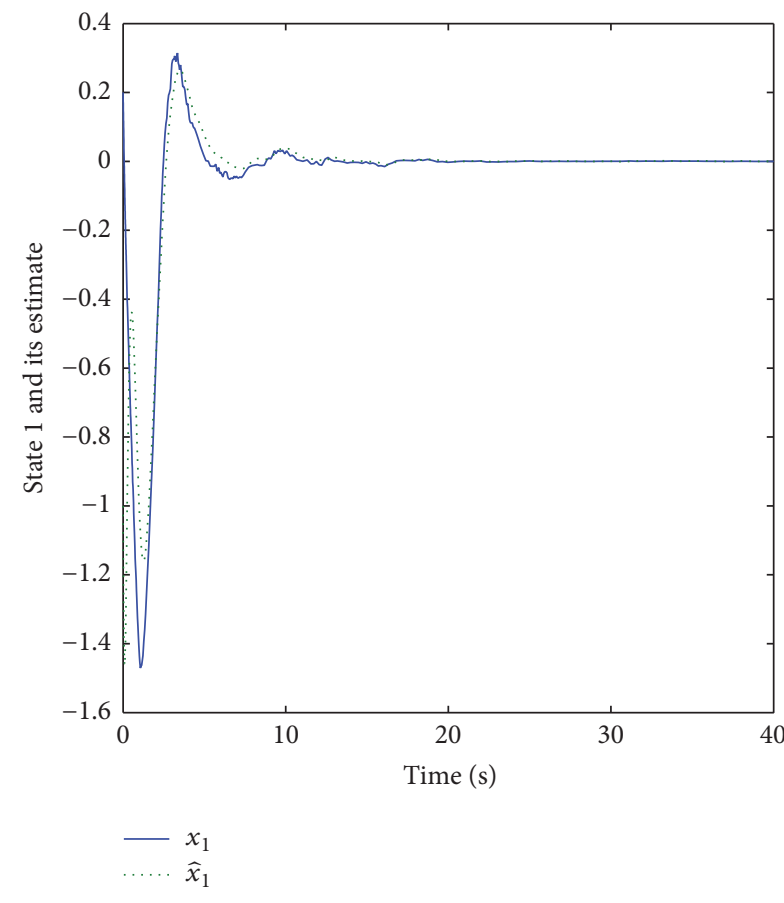

(a)

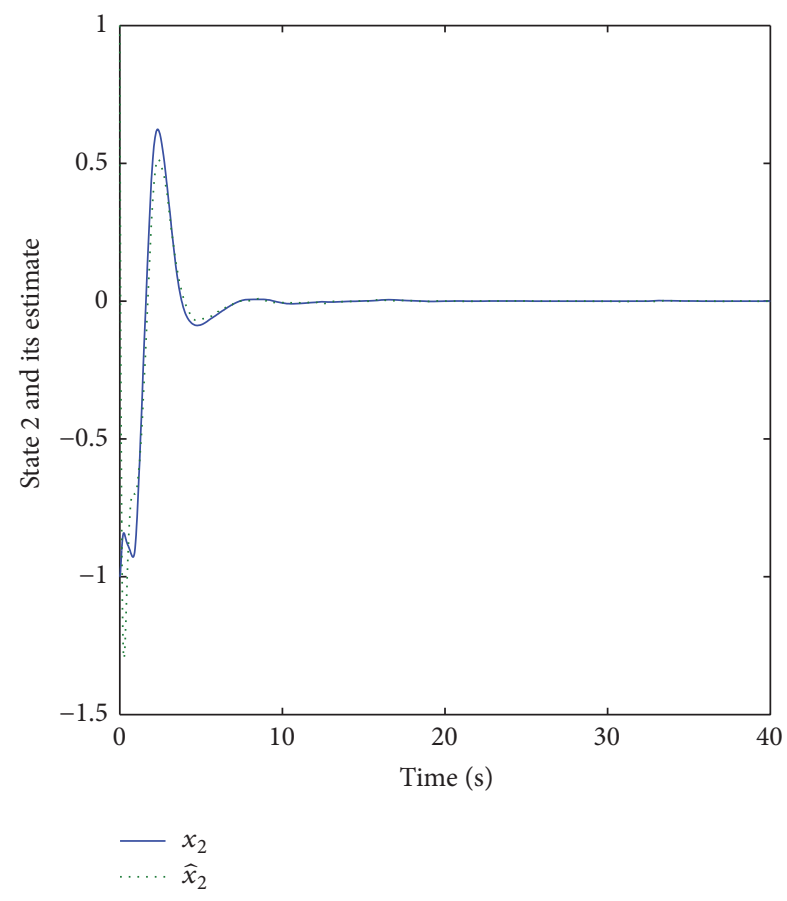

(b)

FIgURE 1: The states and their estimates by RNN.

Figures 1(a) and 1(b) show the states and their estimates (dotted) by RNN for Example 15. It can be clearly seen that all states were estimated. The trajectories of the modeling errors $\left(e_{1}=x_{1}-\hat{x}_{1}, e_{2}=x_{2}-\hat{x}_{2}\right)$ by the RNN are shown in Figure 2, where we can see that the state estimation errors are UUB around the equilibrium. Hence, we can see that the nonlinear system can be well reconstructed by the RNN datadriven model as Theorem 7 predicted.

Based on the obtained data-driven model, the approximation robust NN controller is implemented for the unknown affine nonlinear continuous-time system (49). Parameters for adaptive laws in Theorem 14 are selected as $\delta_{\mathscr{\omega}}=0.0002, \delta_{r}=$ $0.0005, \delta_{\xi_{m}}=0.0002, \omega_{0}=0.5, \sigma_{0}=0.4$. The initial values of the parameters are selected as $\widehat{\omega}(0)=0.1, \widehat{r}(0)=0.5$, $\widehat{\xi}_{m}(0)=0.01$ and the initial values of the states are $x_{0}=$ $[-0.2,-1]^{T}$.

Under the proposed nonlinear adaptive controller scheme (32), the simulation results are shown in Figures 3-5. As shown in Figure 3, the smooth control input is bounded and convergent. It is shown in Figure 4 that the stability of the closed-loop system can be obtained and the state quickly converges to zero for the nonlinear system (49) in the presence of time-varying external disturbances and input saturations. Figure 5 shows the estimated curves of control parameters $\widehat{\omega}, \widehat{r}$, and $\widehat{\xi}_{m}$. All of the four estimation parameters converge to the real values. We can see that the closed-loop system is UUB in the presence of external disturbances and input constraints by the proposed adaptive control algorithm.

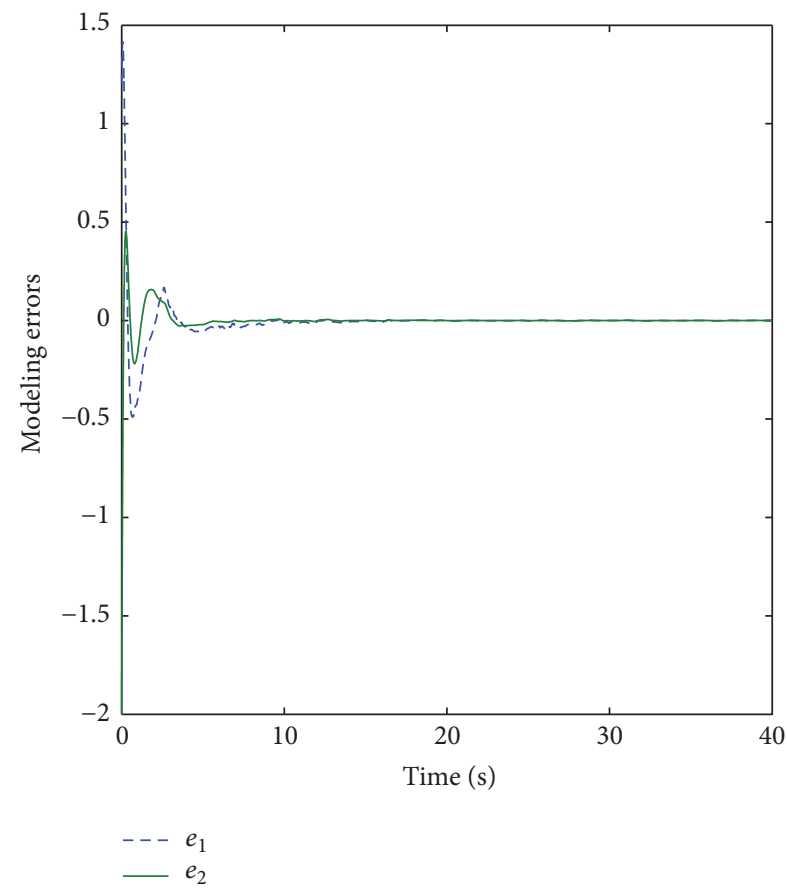

FIgURE 2: The modeling errors by the RNN.

Example 16. Consider that a nonaffine system is described by the following differential equation [30]: 


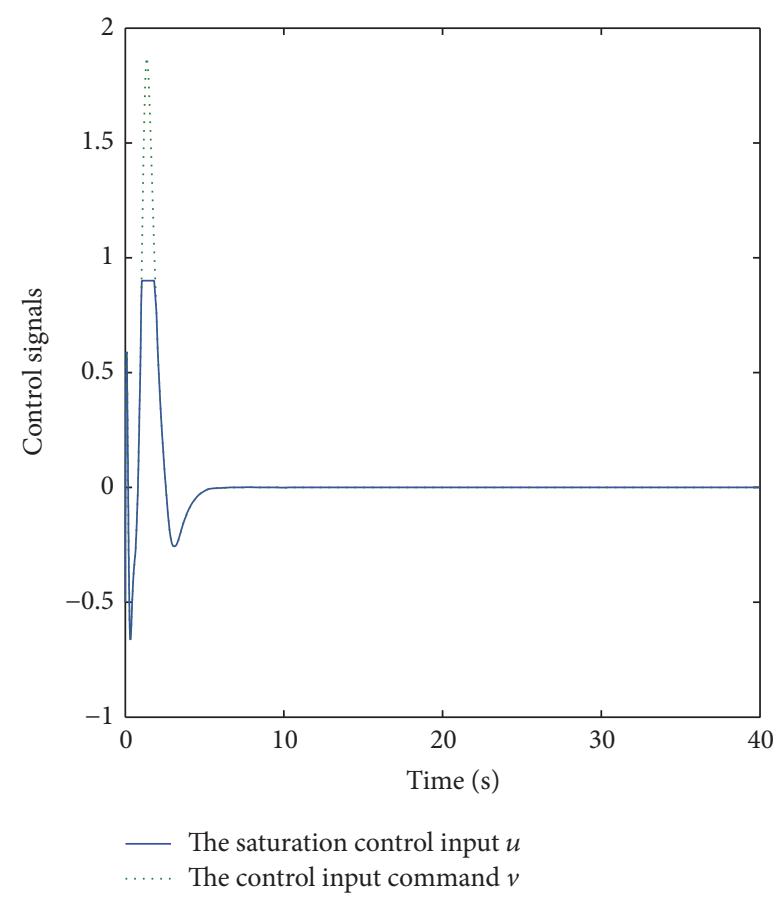

FIGURE 3: Control input.

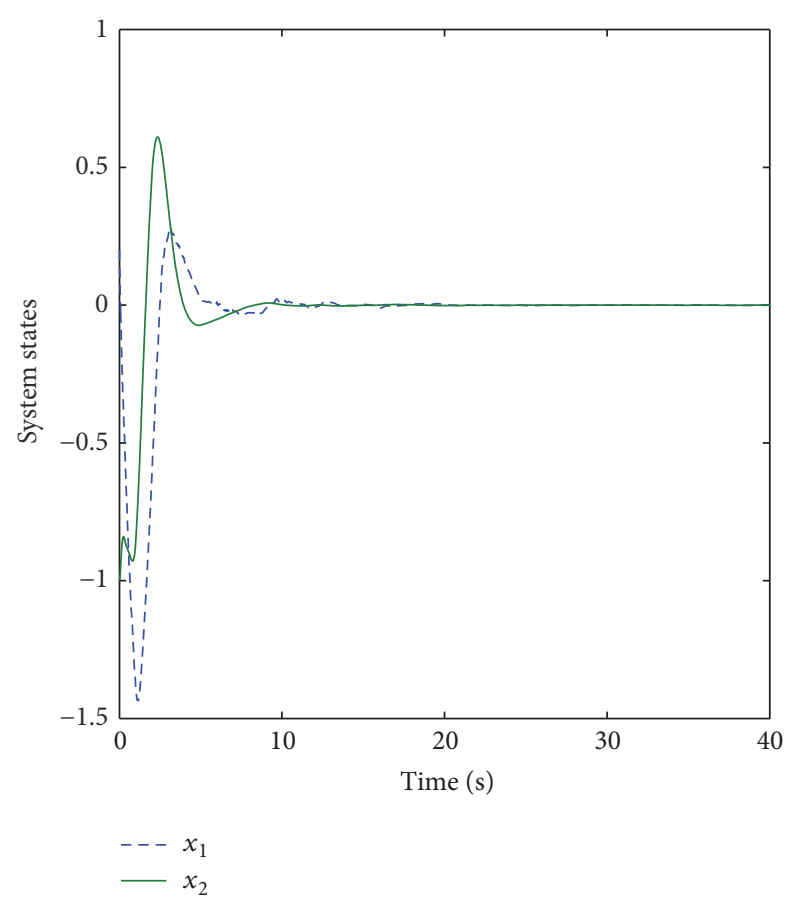

FIgURE 4: The closed-loop system states response.

$$
\begin{aligned}
\dot{x}= & {\left[\begin{array}{c}
x_{2} \\
x_{1}{ }^{2}+0.15 u^{3}+0.1\left(4+x_{2}{ }^{2}\right) u+\sin (0.1 u)
\end{array}\right] } \\
& +\left[\begin{array}{l}
1 \\
0
\end{array}\right] d(t) .
\end{aligned}
$$

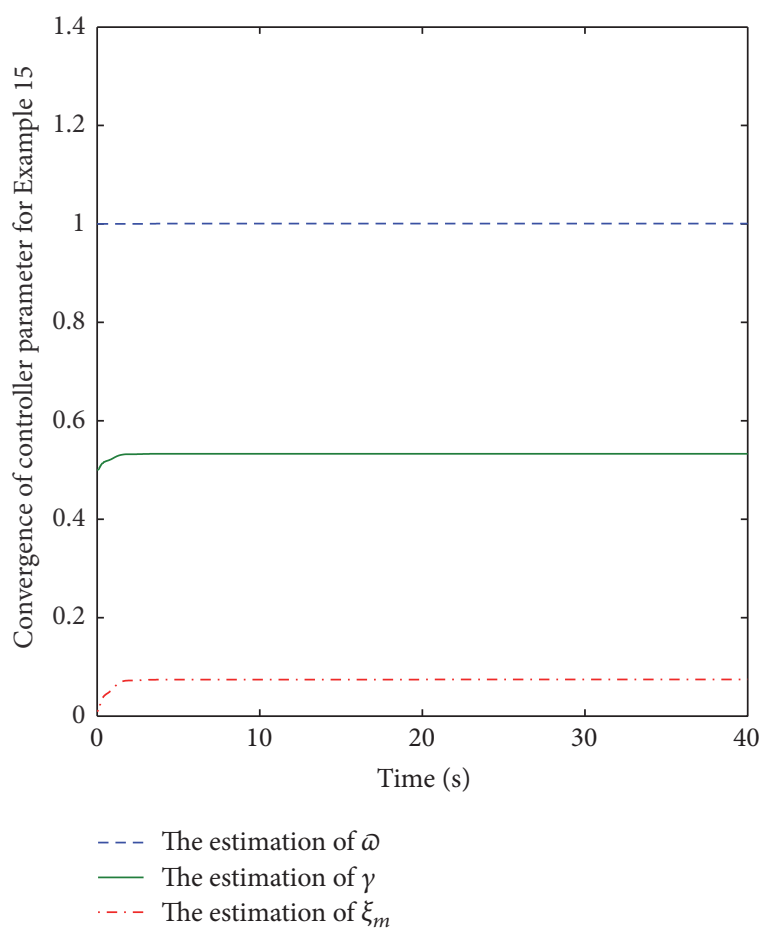

FIgURE 5: Convergence of $\widehat{\omega}, \widehat{\xi}_{m}$, and $\widehat{r}$.

Similarly, to maintain the excitation condition, the external disturbance $d(t)$ as (50) is added to system (51).

Choose the following simulation parameter: $\delta_{\infty}=\delta_{\xi_{m}}=$ $0.002, \delta_{r}=0.001, \omega_{0}=5, \sigma_{0}=0.1, \varepsilon=\left[\begin{array}{llll}-0.1 & 10 ; & -3 & 6\end{array}\right]$ and the initial values are $\widehat{\widehat{\omega}}(0)=0, \widehat{r}(0)=0, \widehat{\xi}_{m}(0)=0.001$, $\left[x_{1}(0), x_{2}(0)\right]=[0.5,-1]$.

Using a similar method as in Example 15, based on the RNN model and the nonlinear system (51), Figures 6(a) and 6(b) show the states and their estimates (dotted). It can be clearly seen that all states were estimated. The curves of the system model errors are shown in Figure 7. It is observed that the obtained data-driven model can reconstruct the unknown nonaffine nonlinear system (51) successfully as Theorem 7 predicted.

Under the proposed nonlinear adaptive controller scheme (32), the simulation results are shown in Figures 8-10 for the nonaffine nonlinear system (51). The smooth bounded control input is convergent as shown in Figure 8. It is shown in Figure 9 that the stability of the closed-loop system can be obtained and the state variables quickly converge to zero for the nonlinear system (51) with time-varying external disturbances and input constraints. Figure 10 shows the evolution curves of control parameters $\widehat{\hat{\omega}}, \widehat{r}$, and $\widehat{\xi}_{m}$, which are bounded. We can see that the closed-loop system is UUB in the presence of external disturbances and input constraints by the proposed adaptive control algorithm.

These simulation results demonstrate the tracking capability of the proposed controller which is valid for nonaffine nonlinear system with the time-varying unknown external disturbance and input saturation and satisfying tracking performance is obtained even for the unknown system dynamics. 


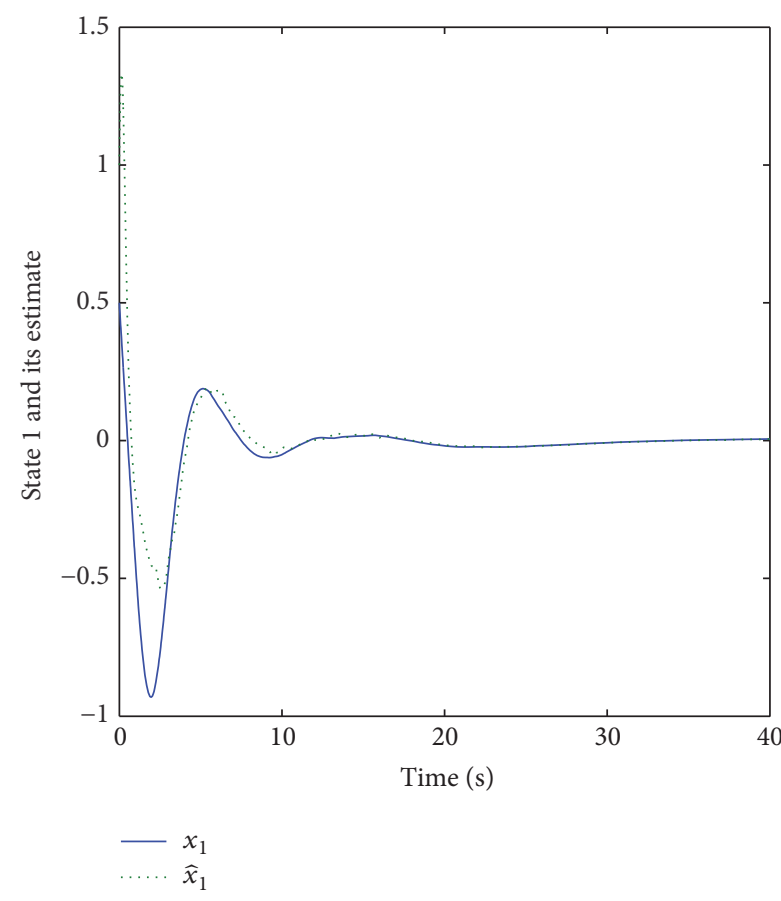

(a)

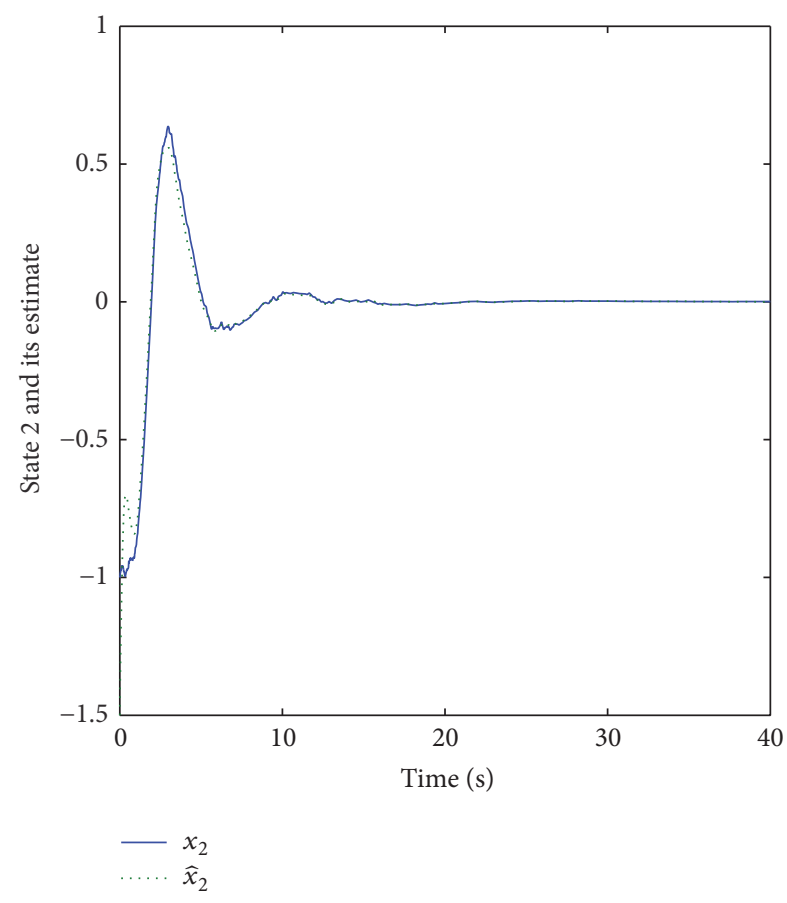

(b)

Figure 6: The states and their estimates by RNN.

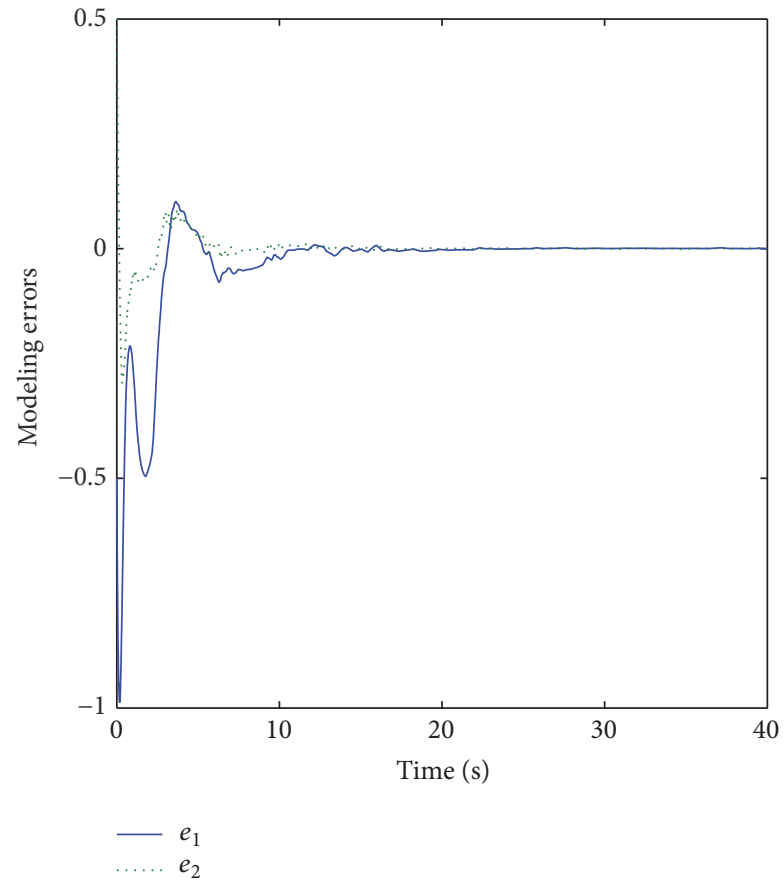

Figure 7: The modeling errors by the RNN.

\section{Conclusion}

In this paper, the data-driven robust control scheme is designed for an unknown nonlinear system with consideration of input constraints nonlinearity and unknown external

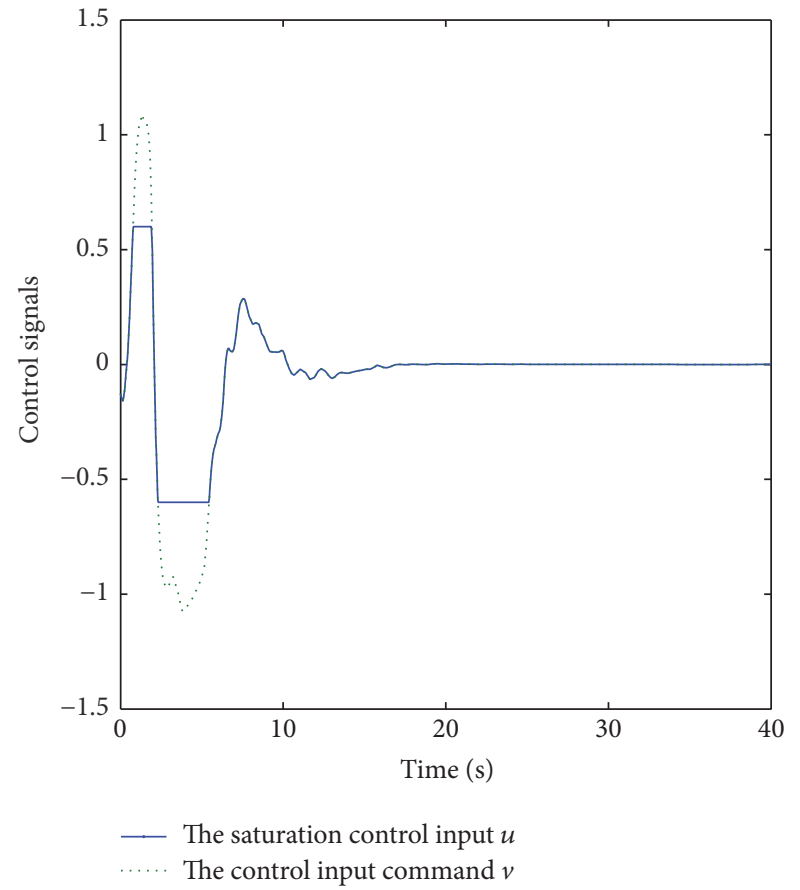

FIgURE 8: Control input.

disturbances. An RNN was employed to reconstruct the nonlinear with data-driven model. Based on the obtained model, an adaptive NN control scheme has been developed to cope with the time-varying disturbances and input saturations. By the proposed adaptive NN control, the UUB of all of the 


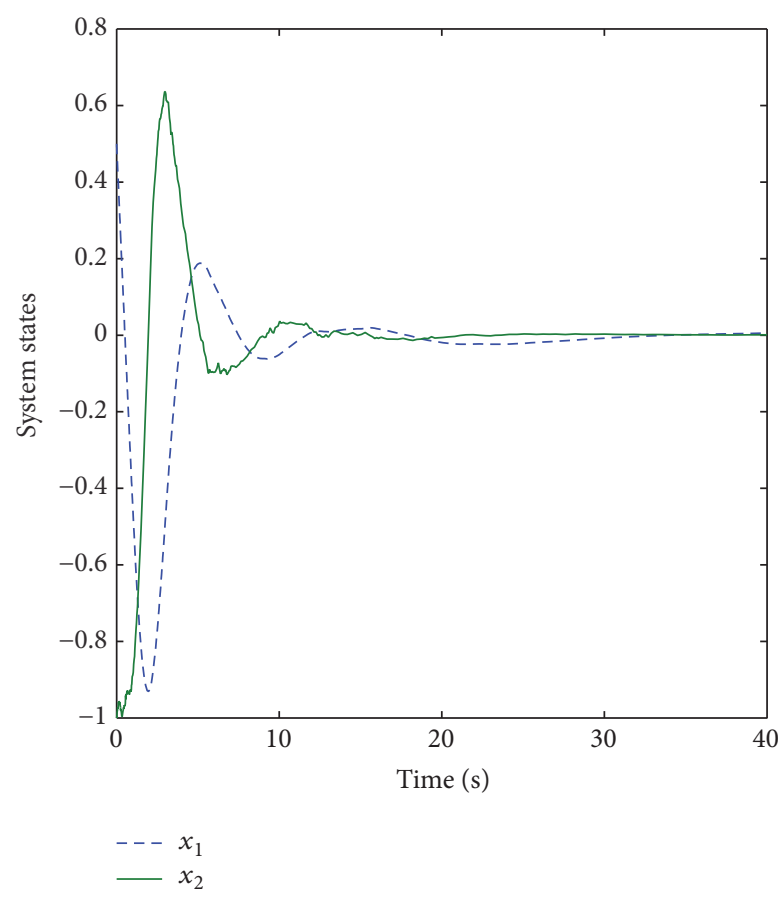

FIGURE 9: The closed-loop system states response.

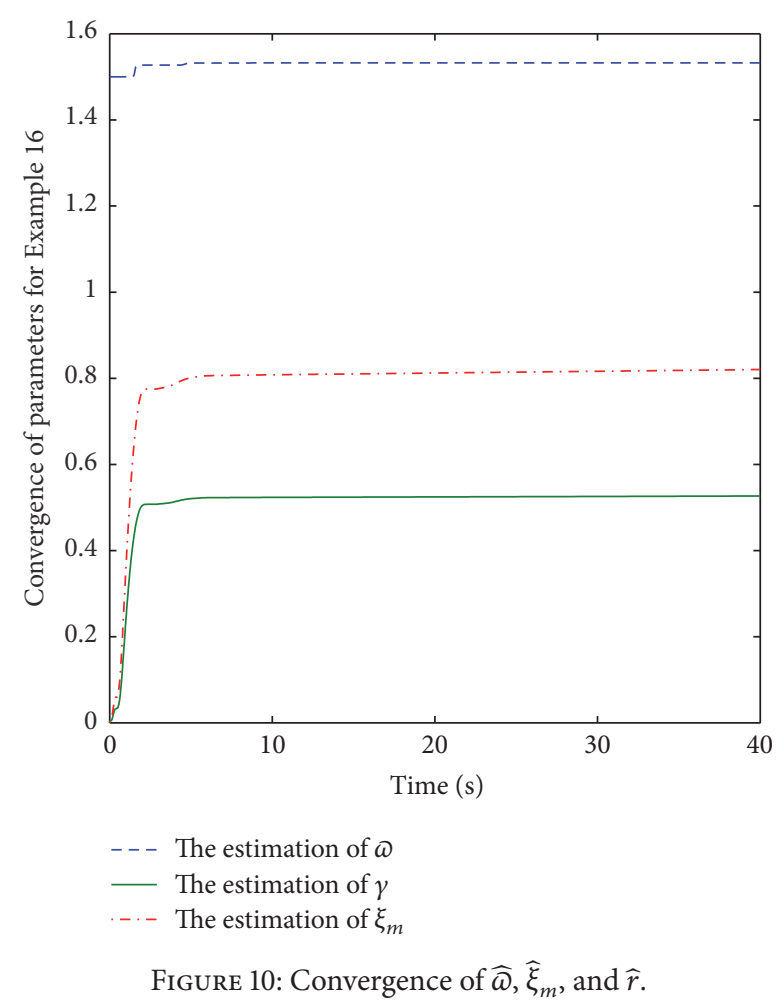

signals of the closed-loop system is guaranteed via Lyapunov approach. Simulation results demonstrate the effectiveness of the proposed algorithm, from which we can find that the good performance is guaranteed. The primary goal of our future work is to devise appropriate conditions and novel parameters updating laws rendering asymptotically stability of modeling error of reconstructed unknown continuoustime nonlinear system. In addition, we continue to study the robustness issues in data-driven control not only consideration of external disturbances but also consideration of sensors and actuators faults.

\section{Conflicts of Interest}

The authors declare that they have no conflicts of interest.

\section{Acknowledgments}

This work is supported by the National Natural Science Foundation of China (61074063 and 61473147).

\section{References}

[1] J. de Jesús Rubio, "Discrete time control based in neural networks for pendulums," Applied Soft Computing, 2017.

[2] Y. Pan, M. J. Er, T. Sun, B. Xu, and H. Yu, "Adaptive fuzzy PD control with stable Ho tracking guarantee," Neurocomputing, vol. 237, pp. 71-78, 2017.

[3] J. de Jesús Rubio, L. Zhang, E. Lughofer, P. Cruz, A. Alsaedi, and T. Hayat, "Modeling and control with neural networks for a magnetic levitation system," Neurocomputing, vol. 227, pp. 113121, 2017.

[4] Y. Pan, Y. Liu, B. Xu, and H. Yu, "Hybrid feedback feedforward: An efficient design of adaptive neural network control," Neural Networks, vol. 76, pp. 122-134, 2016.

[5] D. G. Ward and J. F. Monaco, "System identification for retrofit reconfigurable control of an F/A-18 aircraft," Journal of Aircraft, vol. 42, no. 1, pp. 63-72, 2005.

[6] C. Tan, G. Tao, and R. Qi, "An adaptive control scheme using multiple reference models," International Journal of Adaptive Control and Signal Processing, vol. 28, no. 11, pp. 1290-1298, 2014.

[7] M. Chen and W.-H. Chen, "Disturbance-observer-based robust control for time delay uncertain systems," International Journal of Control, Automation and Systems, vol. 8, no. 2, pp. 445-453, 2010.

[8] J. Zhao, B. Jiang, F. N. Chowdhury, and P. Shi, "Active faulttolerant control for near space vehicles based on reference model adaptive sliding mode scheme," International Journal of Adaptive Control and Signal Processing, vol. 28, no. 9, pp. 765777, 2014.

[9] R. K. Lim and M. Q. Phan, "Identification of a multistep-ahead observer and its application to predictive control," Journal of Guidance, Control, and Dynamics, vol. 20, no. 6, pp. 1200-1206, 1997.

[10] J. M. Lee and J. H. Lee, "Approximate dynamic programmingbased approaches for input-output data-driven control of nonlinear processes," Automatica. A Journal of IFAC, the International Federation of Automatic Control, vol. 41, no. 7, pp. 12811288, 2005.

[11] A. Lecchini, M. C Campi, and S. M Savaresi, "Virtual reference feedback tuning for two degree of freedom controllers," International Journal of Adaptive Control \& Signal Processing, vol. 16, no. 5, pp. 2416-2421, 2002.

[12] J. D. J. Rubio and W. Yu, "Stability analysis of nonlinear system identification via delayed neural networks," IEEE Transactions on Circuits and Systems II: Express Briefs, vol. 54, no. 2, pp. 161$165,2007$. 
[13] H. Zhang, L. Cui, X. Zhang, and Y. Luo, "Data-driven robust approximate optimal tracking control for unknown general nonlinear systems using adaptive dynamic programming method," IEEE Transactions on Neural Networks, vol. 22, no. 12, pp. 2226-2236, 2011.

[14] Q. Wei, R. Song, and P. Yan, "Data-driven zero-sum neurooptimal control for a class of continuous-time unknown nonlinear systems with disturbance using ADP," IEEE Transactions on Neural Networks and Learning Systems, vol. 27, no. 2, pp. 444458, 2016.

[15] F. Wang, Q. Zou, C. Hua, and Q. Zong, "Disturbance observerbased dynamic surface control design for a hypersonic vehicle with input constraints and uncertainty," Proceedings of the Institution of Mechanical Engineers. Part I: Journal of Systems and Control Engineering, vol. 230, no. 6, pp. 522-536, 2016.

[16] L. Lu and B. Yao, "Online constrained optimization based adaptive robust control of a class of MIMO nonlinear systems with matched uncertainties and input/state constraints," Automatica. A Journal of IFAC, the International Federation of Automatic Control, vol. 50, no. 3, pp. 864-873, 2014.

[17] M. Chen, G. Tao, and B. Jiang, "Dynamic surface control using neural networks for a class of uncertain nonlinear systems with input saturation," IEEE Transactions on Neural Networks and Learning Systems, vol. 26, no. 9, pp. 2086-2097, 2015.

[18] J. Ma, S. S. Ge, Z. Zheng, and D. Hu, "Adaptive NN control of a class of nonlinear systems with asymmetric saturation actuators," IEEE Transactions on Neural Networks and Learning Systems, vol. 26, no. 7, pp. 1532-1538, 2015.

[19] Q. Zhou, P. Shi, Y. Tian, and M. Wang, "Approximation-Based Adaptive Tracking Control for MIMO Nonlinear Systems with Input Saturation," IEEE Transactions on Cybernetics, vol. 45, no. 10, pp. 2119-2128, 2015.

[20] Z. Chen, Z. Li, and C. L. P. Chen, "Adaptive Neural Control of Uncertain MIMO Nonlinear Systems With State and Input Constraints," IEEE Transactions on Neural Networks and Learning Systems, 2016.

[21] J. de Jesús Rubio, "Sliding mode control of robotic arms with deadzone," IET Control Theory \& Applications, vol. 11, no. 8, pp. 1214-1221, 2017.

[22] C. Aguilar-Ibanez, H. Sira-Ramirez, and M. S. SuarezCastanon, "A linear active disturbance rejection control for a ball and rigid triangle system," Mathematical Problems in Engineering, Article ID 1358930, Art. ID 1358930, 11 pages, 2016.

[23] J. D. J. Rubio, "Hybrid controller with observer for the estimation and rejection of disturbances," ISA Transactions, vol. 65, pp. 445-455, 2016

[24] E. Lavretsky, "K A Wise, Robust and Adaptive Control," Advanced Textbooks in Control Signal Processing, vol. 11, no. 96, pp. 367-372, 2013.

[25] M. M. Polycarpou, "Stable adaptive neural control scheme for nonlinear systems," Institute of Electrical and Electronics Engineers. Transactions on Automatic Control, vol. 41, no. 3, pp. 447451, 1996.

[26] S. S. Ge and C. Wang, "Adaptive neural control of uncertain MIMO nonlinear systems," IEEE Transactions on Neural Networks, vol. 15, no. 3, pp. 674-692, 2004.

[27] R. Enns and J. Si, "Helicopter trimming and tracking control using direct neural dynamic programming," IEEE Transactions on Neural Networks, vol. 14, no. 4, pp. 929-939, 2003.

[28] S. Mohammad-Hoseini, M. Farrokhi, and A. J. Koshkouei, "Robust adaptive control of uncertain non-linear systems using neural networks," International Journal of Control, vol. 81, no. 8, pp. 1319-1330, 2008.

[29] H. Chen, Y. Gong, and X. Hong, "Online modeling with tunable RBF network," IEEE Transactions on Systems Man and Cybernetics Part B Cybernetics A Publication of the IEEE Systems Man and Cybernetics Society, vol. 43, no. 3, pp. 935-947, 2013.

[30] S. Doudou and F. Khaber, "Adaptive fuzzy control of a class of SISO nonaffine nonlinear systems," in Proceedings of the 1st Mediterranean Conference on Intelligent Systems and Automation, CISA '08, pp. 103-108, dza, July 2008. 


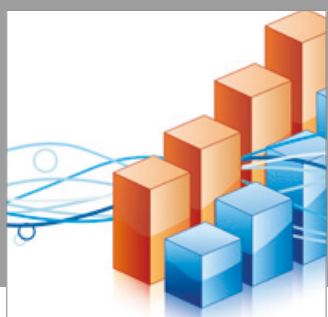

Advances in

Operations Research

vatersals

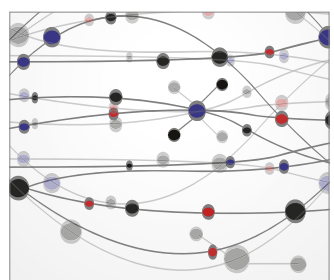

\section{The Scientific} World Journal
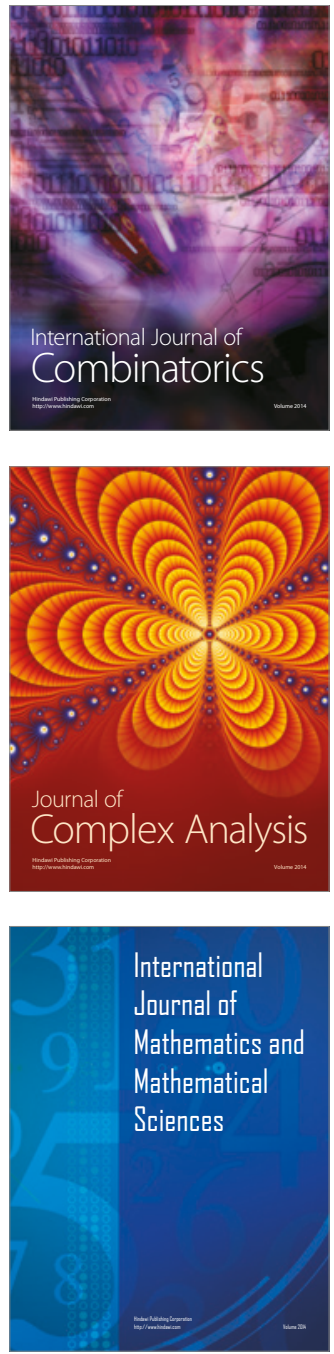
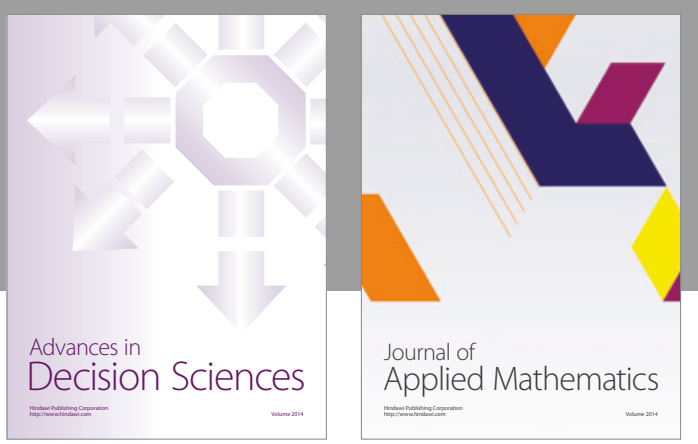

Algebra

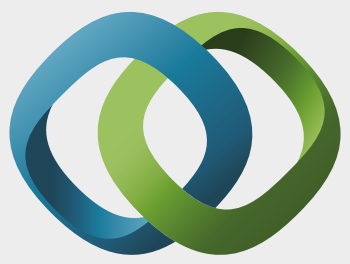

\section{Hindawi}

Submit your manuscripts at

https://www.hindawi.com
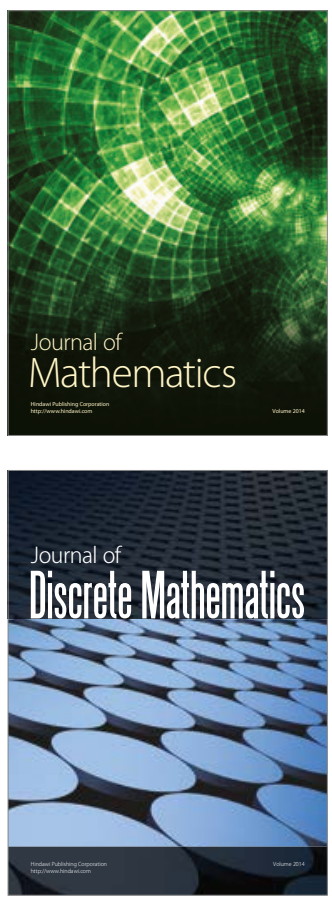

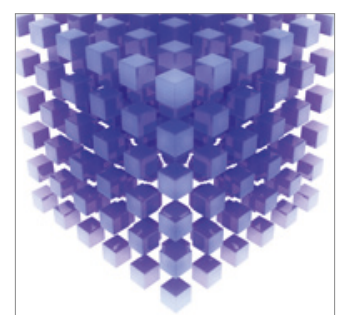

Mathematical Problems in Engineering
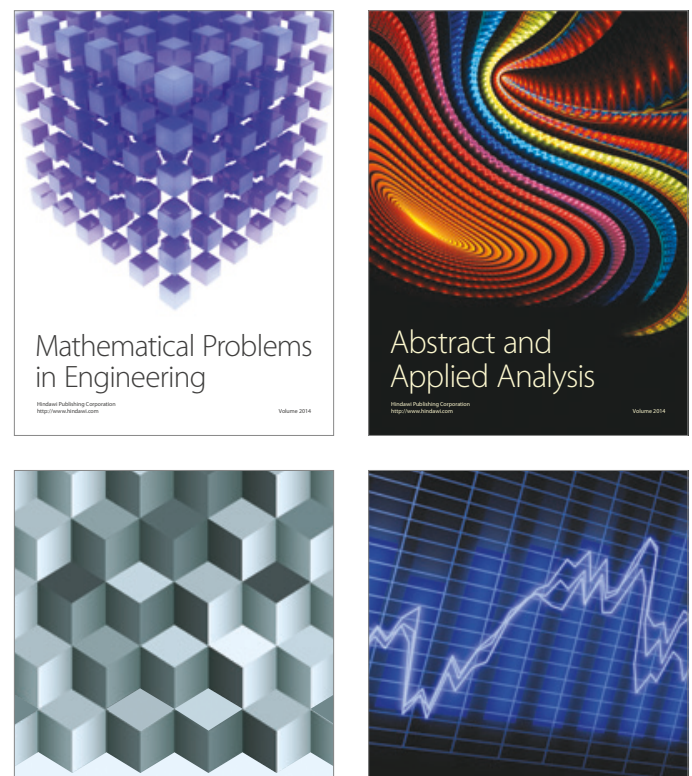

Journal of

Function Spaces

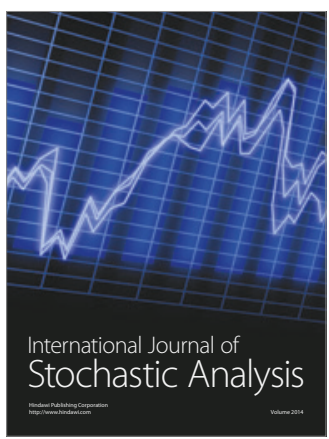

Probability and Statistics
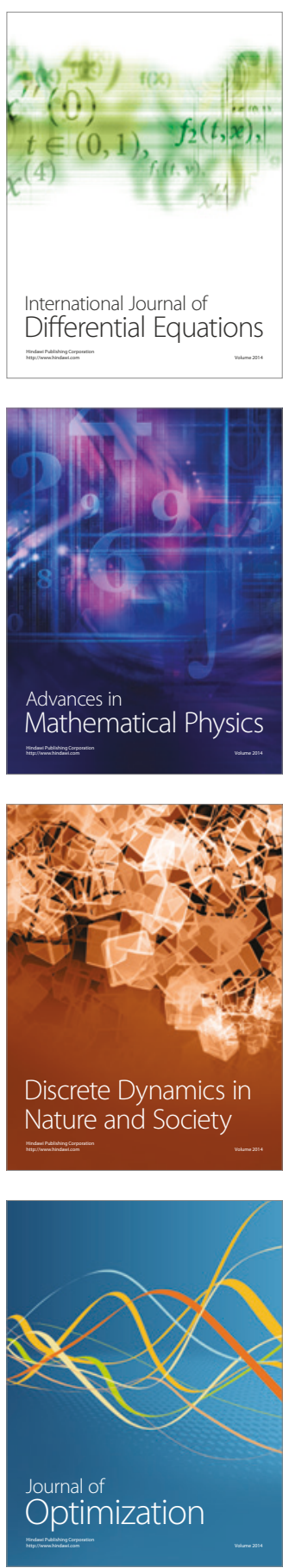